\title{
English Writing Program for Students of Science and Technology
}

\begin{abstract}
ZHANG Zhen
Leshan Normal University, Leshan, China

General college English education cannot face the urgency for the future scientific and technological professionals to make their voices in the international arena. This paper introduces the English writing program which aims at improving college students' English technical writing proficiency so that they can write clear, concise, and effective English.

Keywords: technical writing program, syllabus design, instructional approaches, writing proficiency
\end{abstract}

\section{Introduction}

As our current world has entered the era of international communication and advanced technology, there are more and more chances for engineers and technical professionals to convey technical information in English for various purposes. Therefore, besides learning general English as an international language, students of science and technology of colleges and technical institutes need to be equipped with adequate writing ability so that they can communicate technical information clearly on at least a basic level. A good English writing proficiency can be a contributing factor to their professional recognition and career prospect.

This paper proposes a pragmatic English writing program for students of science and technology of colleges and technical institutes with intermediate ESL (English as a Second Language) proficiency. It can be used as a writing course or as part of a general English course. The program covers a syllabus design and instructional approaches on basic writing skills with particular reference to technical writing. Georges (1996) explained that the purposes of technical writing are to inform and persuade; the subjects are things and development; and its characters are direct, objective, and specific. In short, this is a program which aims to train students in writing clear, concise, and effective English.

\section{Syllabus Design}

This program consists of four stages, which include: preparation, tool acquisition, summary writing, and composition.

\section{Preparation Stage}

This is the stage for building up students' confidence or reshaping their perceptions of English writing. Some students of science and technology may think that English writing is a very difficult task; yet they can still write comprehensibly if they know how to apply the basic grammar rules and make use of simple and

ZHANG Zhen, senior lecturer, doctoral candidate, School of Foreign Languages and Literature, Leshan Normal University, Leshan, China. 
concrete words. The ability to express their ideas in clear and understandable English is something they can acquire systematically.

To start with, ask the students to write a few short essays on topics they are very familiar with, such as self introduction, description of a family member, a close friend, daily living patterns, or their own chosen topic. Chose a topic in which they have concrete content to write about. A few guidelines and vocabularies may be provided if students are of a low-intermediate level. Encourage them to use the words and structures they are familiar with. The writing process at this stage serves as a warm-up exercise. Let the students work on something they can easily handle as a way to help them experience some competence in freely expressing themselves. For the revision part, leave the common errors for the students to correct themselves by giving indicative symbols or abbreviations and revise those awkward expressions. Respond to the content and write encouraging remarks whenever possible.

\section{Tools Acquisition Stage}

Many grammatical constructions are important for writing good English. The principle of this stage, however, is to let the students first get familiar with a few basic essential tools that can enhance their sentence clarity in technical writing. Other relevant constructions can be reviewed if time is available.

The use of subordinate clauses. Technical writing involves a lot of analytic writing. Subordinate clauses are powerful tools for analyzing the logical relationships among ideas such as a chronological development, a cause-effect relationship, a purpose relationship, and a condition relationship.

Students can get familiar with this kind of construction by the following steps. First, briefly explain the meaning of a subordinate clause. Then give the students a list of complex sentences. Ask them to identify the subordinate clauses and the subordinating conjunctions as well as to explain the relationship expressed in each sentence. The next step is practice. Ask the students to combine two sentences or a set of isolated ideas into one sentence by using appropriate subordinating conjunctions. For example:

(1) New racing bicycles have disc wheels. Wind resistance is reduced (E. H. Glendinning \& N. Glendinning, 1995).

(2) Copper is highly conductive. It is used for electric wiring (E. H. Glendinning \& N. Glendinning, 1995).

(3) The water is heated. It reaches a pre-set temperature (E. H. Glendinning \& N. Glendinning, 1995).

(4) The program was written in FORTRAN.

(5) It is longer than a machine language version.

(6) It can run on many different computers (Greaney, 1997).

(7) Eunice set fire to the cat. The cat jumped into the pool.

(8) The cat could not swim. Rodney rescued the cat (Greaney, 1997).

Finally, ask them to make up their own complex sentences.

The use of relative clauses. Technical writing often involves detailed definition or modification of a particular term. Relative clauses can be useful tools for defining and qualifying nouns instead of putting a long list of technical nouns or adjectives before a noun. In that case, the sentence can be less confusing. The "where" clause is also useful for describing the location of stages described in a process.

Similarly, give students a list of sentences that contain clauses introduced by a relative pronoun. Ask the students to identify the relative clauses and explain their functions. Examples of restrictive and nonrestrictive clauses should be included for the students to find out how differently they function. The following type of sentence completing exercise can help the students understand and apply relative clauses, for example: 
(9) Students are more likely to get good grades.

(10) Aluminum, is used to make aircraft.

(11) The new method, is faster than the conventional method.

(12) We like to live in areas

Introduce the possibility of replacing relative clauses by infinitives or participles after the students have become acquainted with relative clauses.

The use of parallelism. Parallelism is the principle that units of similar content and function should be expressed in equal form. Repetition of the same structure allows the reader to recognize the ideas more readily. Long sentences can be made clearer and easier to understand if they are grammatically parallel. This is also a useful tool when writing comparison and contrast. Illustrate the concept of parallelism by giving a list of sample sentences, which use different grammatical constructions like prepositions, conjunctions, clauses, and infinitives to make similar ideas parallel. Then ask the students to practice by rewriting sentences parallel. For example:

(13) We are looking for engineers with realistic decision-making capabilities, who can think logically, and who have the ability to analyze situations carefully (Neufeld, 1987).

(14) It is necessary to communicate well for business, but industry has its needs too, and government reports and proposals must be well written (Neufeld, 1987).

(15) Advances in technology have made our life more convenient, comfort has been added to our lives, and safety is also important (Neufeld, 1987).

The use of comma and semicolon. Correct uses of comma can eliminate sentence confusion and avoid misinterpretation of the intended meaning. Highlight some common rules of using commas as well as the use of semicolons in separating two closely related independent clauses in a compound sentence and in separating very long phrases or sentences where the use of comma will lead to ambiguity. Illustrate the uses with examples.

\section{Summary Writing Stage}

This stage serves several purposes. First, it works as a transition stage for the subsequent composition stage. When students write summaries, they can focus more on the practice of language skills without worrying too much on the content part, provided that the chosen text is not too difficult to comprehend. In addition, the chosen texts can also serve as sort of model writing, grammar guides, and sources of new vocabulary. Second, summary writing requires good skills on concise writing; otherwise, only a small portion of the original ideas can be expressed in a limited number of words. Third, it is appropriate to introduce paragraph characteristics at this stage as the students have to start writing in paragraphs.

Before they start writing summaries, introduce the following techniques.

Conciseness. This is a world of information explosion. The reader has so much to read. Efficiency and conciseness in informative writing can save personal time and social cost. Concise writing is a skill, which can be achieved by applying the general guidelines into the writing practice. They: (1) delete redundant and unnecessary material; (2) reduce clauses and phrases to shorter constructions like an adjective phrase or a single word without losing the needed emphasis; (3) eliminate any words or phrases that can be removed without damaging the meaning of the sentence or paragraph; and (4) illustrate the general guidelines with example phrases and sentences. Let the students notice the differences between wordy and concise phrases. Then they can do some exercises on rewriting concise phrases and sentences. 
Unity and coherence. Another learning focus of this stage is to understand paragraph characteristics like unity and coherence. A paragraph is unified if every sentence in the paragraph is relevant to the general topic of that paragraph. All sentences should be logically related in one paragraph. Coherence is achieved by using transition words or phrases, pronouns that refer to nouns in previous sentences, or repetition of words previously mentioned. Illustrate the effects of coherence by comparing example paragraphs with coherence destroyed with the ones that are written coherently. Then ask the students to fill in appropriate transition words missed in a given text as a practice.

How to write a summary. Students tend to copy sentences exactly from the original article and put them together when they write summaries. In that case, they will lose the opportunity in fully utilizing their practice of writing skills. Furthermore, it is unlikely that they can include all the important ideas mentioned. A summary should be clear and balanced so that the reader can grasp the main ideas of the original article without reading it. A better way to make a summary is to jot down the main points in note form and then link them clearly into concise sentences in the students' own words. Following that, link the sentences into coherent paragraphs. Suggest the students to write a topic sentence for each paragraph so as to help them stay focused on the main idea of that paragraph. Finally, edit their work and check if the number of words used is within the stated limit. A suggested number for beginners is $150-200$ words.

Greaney (1997) found that students wrote more focused summaries with more complex sentence structure than they had used in their earlier, longer summaries on the request of writing the one-sentence summary. As a practice of more complex and syntactically sophisticated sentences, students may try to write very short summaries approaching the end of this stage.

\section{Composition Stage}

This is the stage where students practice some common patterns of technical writing with the application of skills taught at previous stages. Topics appropriate to their proficiency and background are suggested for each type of writing. Students can share some ideas and vocabulary during group discussion as a pre-writing activity.

To write definitions of objects. One major method of organizing technical writing is definition, which includes the single-sentence definition and the extended definition. The single-sentence definition defines an object by giving the category and the distinguishing characteristics and it is useful in defining something concrete. Students can practice the one-sentence definition on a tool or machine that is frequently used, for instance: a computer, dictionary, watch, electronic calculator, telephone, or fax machine by using the following schema.

(16) is a kind /form/category/device/type of (class) that (distinguishing feature).

They can also add extra information to write an extended definition. Strategies used to write extended definitions are use of examples, analogies, description of physical characteristics, components, and functions. Some suggested topics for extended definition are electricity, television, electric coffee pot, refrigerator, microwave oven, and building.

To write a process. A process is written to instruct or to inform. A clear description of a process involves sequencing the stages, locating the stages, describing what happens at each stage, and explaining what happens at each stage. Most operational process descriptions follow a chronological order, which can be shown by using sequence words like first, next then, subsequently, at this point, later, afterwards, finally and so on. The stages 
can be located by using a prepositional phrase "in/at the..." or in some cases using a "where" clause to link a stage, its location, and what happens there. For example:

(17) The warm gas passes through the condenser, where it heats the surrounding and cools down (E. H. Glendinning \& N. Glendinning, 1995).

For the part in describing and explaining what happens in each stage, think of the answers to "What happens? Why? How?".

Here are some suggested topics for writing a process:

(1) To describe the process of getting cash from an automatic teller machine

(2) To describe the process of sending or retrieving an e-mail

(3) To describe the process of performing a laboratory practice

(4) To describe the process of fixing a piece of DIY furniture

(5) To describe the process of changing components of appliances or vehicles

(6) To describe the process of operating the washing machine

(7) To describe the process of arranging a vacation trip

To write comparison and contrast. We use this type of writing to relate the similarities and differences among things. This writing technique can be applied when it is necessary to compare and contrast different proposals, solutions to problems, and improvement or design change of a conventional product with a new product.

Students can organize a compare-contrast paper in two ways: the point-by-point development and whole-by-whole method. In a point-by-point development, each point is contrasted. On the other hand, the whole-by-whole method utilizes entire blocks of information about each topic. When using the whole-by-whole method, keep the main order of points the same for each idea compared or contrasted to avoid splitting the paper into separate compositions without discernible themes. Use transitions that constantly refer back to the first idea being compared or contrasted. Remind the students to use the comparative form of the adjective or adverb, expressions like but, whereas, in contrast, different from, unlike... to describe differences; and words and phrases like similarly, likewise, in the same way... to describe similarities.

Here are some suggested topics for writing comparison and contrast:

(1) To compare and contrast two sets of information presented in a chart or graph

(2) To compare and contrast people's life nowadays and 10 years ago

(3) To compare and contrast the living environment of two residential areas

(4) To compare and contrast two outing/class function proposals

(5) To compare and contrast two English learning methods/study plans

(6) To compare and contrast two generations of a product, e.g., computer/stereo/robot

(7) To compare and contrast two brand names of a product

For instance, cellular phone, sports equipment, electronic dictionary, bicycles and so on.

To write analysis. Expository writing is used to explain and clarify. Technical writing is, to a large extent, writing used to convince someone of something. A more effective approach to convince others is to support the broad general statements with details, i.e., begin with a generalization or topic sentence that covers the whole topic and then substantiate the generalization at a more specific level with facts, examples, statistics, and other details. Students can begin by writing two to three sentences to support the stated generalization. For example: 
(18) There are several linguistic factors that make it difficult for a foreign student to learn English. First, ...; Second, ... (Blanton, 1993).

(19) It is difficult for foreign students to adjust to life in a new country for several reasons. First,...; Second, ... (Blanton, 1993).

Then try to write a more detailed analysis on the following suggested topics:

(1) Analyze the reasons for an engineering student to study English

(2) Analyze the effects of the internet to the general public

(3) Analyze the causes of a TV program/movie/song for being very popular

(4) Analyze the adjustments of a role change

(5) Analyze the qualities of an ideal college/teacher/spouse

(6) Analyze the consequences of an important school/government policy

(7) Analyze the problems faced by a new immigrant in your country

Two more techniques can be introduced sometime during this stage for more effective writing. Choose some example sentences from the students' composition and ask them to discuss and suggest corrections.

Use active verbs whenever possible. Active verbs make the sentence stronger and give force to the writing. On the other hand, passive voice constructions are less direct, less natural, and less vigorous. Use the passive voice only when it is needed.

Technical content in an English manuscript should be communicated in a simple and direct manner so that the readers can reach the author's intended meaning quickly. Use simple, direct, and concrete words whenever possible. Avoid pompous words and words loaded with positive or negative emotions. The use of precise words for intended meaning can enhance clear and direct communication.

\section{Conclusion}

The ability to write clear, concise, and effective English is a valuable asset to students. This paper proposes a writing program to help students of science and technology develop such kind of ability through a stage by stage approach. The students first build up their confidence in English writing at the preparation stage and then they learn some useful tools to write clear and comprehensible sentences at the tools acquisition stage. Following that, they learn to write concisely and coherently at the summary writing stage. Finally, they learn to write effectively on some common patterns of technical writing at the composition stage.

Good writing requires a lot of practice. It is unrealistic to expect great writers after taking a semester or year course. Moreover, the basic techniques taught can serve as a foundation for further training or self training, should there arise a need for frequent technical writing in their future career.

\section{References}

Blanton, L. L. (1993). Composition practice (Book 4, 2nd ed.). USA: Heinle \& Heinle Publishers.

Georges, T. M. (1996). A course in analytical writing for science and technology. Retrieved from http://www.mspiggy.etl.noaa.gov/write/

Glendinning, E. H., \& Glendinning, N. (1995). Oxford English for electrical and mechanical engineering. UK: Oxford University Press.

Greaney, G. L. (1997). Less is more: Summary writing and sentence structure in the advanced ESL classroom. The Internet TESL Journal, 3(9). Retrieved from http://iteslj.org/Techniques/Greaney-Writing.html

Neufeld, J. K. (1987). A handbook for technical communication (pp. 65-68). USA: Prentice-Hall, Inc. 